\title{
3D rolling processing analysis by the Fast Multi-pole Boundary Element Method
}

\author{
G. Shen ${ }^{1}$, D. Liu $^{2}$, C. Yu ${ }^{3} \&$ Y. Zheng ${ }^{4}$ \\ ${ }^{1}$ National Engineering Research Center of Cold Strip Rolling Equipment \\ and Technology, Yanshan University, China \\ ${ }^{2}$ BeiJing Double Fit Machinery and Electrical Equipment Co., Ltd, China \\ ${ }^{3}$ College of Science, Yanshan University, China \\ ${ }^{4}$ College of Mechanical Engineering, Yanshan University, China
}

\begin{abstract}
For a roll gap of continuous plastic forming, the determination of mechanical parameters of a rolling processing involves a multi-body elasto-plastic frictional contact problem. As one of the three important numerical analytic methods, the Boundary Element Method (BEM) is suitable for the solution of contact problems, and it shows superiority to the Finite Element Method (FEM) and Finite Difference Method (FDM) in these cases. However, when the contact objects become very complicated and large-scale discrete nodes are generated, there are inherent difficulties for the BEM, such as time-consuming problem, low efficiency, and so on. To solve these problems, a kind of Fast Multi-pole Boundary Element Method (FM-BEM) is proposed. Combining the Fast Multipole Method (FMM) with BEM opens up a new computational situation, especially when a high efficient solver named Generalized Minimal Residual Algorithm (GMRES (m)) is introduced. Then a node-to-surface frictional contact model and a programming-iteration algorithm are developed. On a PVM network parallel platform, the cold rolling process of 2030 four-high mill with a width-tothickness ratio reaching 1850 is successfully simulated. The total freedom is 18414 and the CPU time is 42 hours and 24 minutes. For this rolling problem, both the high precision and the high computational efficiency are impossible for other numerical analytic methods.
\end{abstract}

Keywords: $3 D$ rolling process, BEM, FMM, GMRES(m), PVM. 


\section{Introduction}

The Fast Multi-pole Method (FMM) originates from a large scale fast approximate calculation in the electrostatic field, and it has become one of the popular fast computational methods. When the FMM is introduced in the Boundary Element Method (BEM) [1], the fundamental kernel solutions are decomposed into special forms. Combined with a Generalized Minimal Residual (GMRES) [2] algorithm, a kind of Fast Multi-pole Boundary Element Method (FM-BEM) is established, which is suitable for the requirement of $3 \mathrm{D}$ rolling process simulation $[3,4]$ and other large scale computations.

Three models have been used in the study of strip rolling process simulation, which includes a 2-D rolling process model, 3D rolling process model and striproll contact process model. The establishment process of the strip-roller contact processing model undergoes four stages. Firstly, a model with artificial friction force at the contact arc is built. Secondly, the Finite Element Method (FEM) is introduced in the friction elements. Thirdly, the Finite Strip Method (FSM) is used to build an elastic-plastic process model for the contact of four-high mill's strip, working roll and back-up roll. Finally, artificial assumptions for the contact pressure between the rollers are cancelled out, which makes it possible to accurately simulate the cold rolling process of a four-high mill.

\section{Mechanical model of the cold strip rolling process}

For the mechanical model of the cold strip rolling process, the information of surface force and deformation in the rolling deformation area can be computed by the equilibrium equation about the problem of strip elastic-plastic continuous molding with rotating rolls (as is shown in Fig. 1).

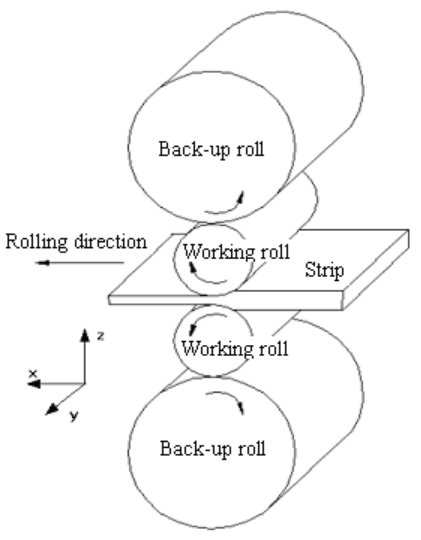

(a)

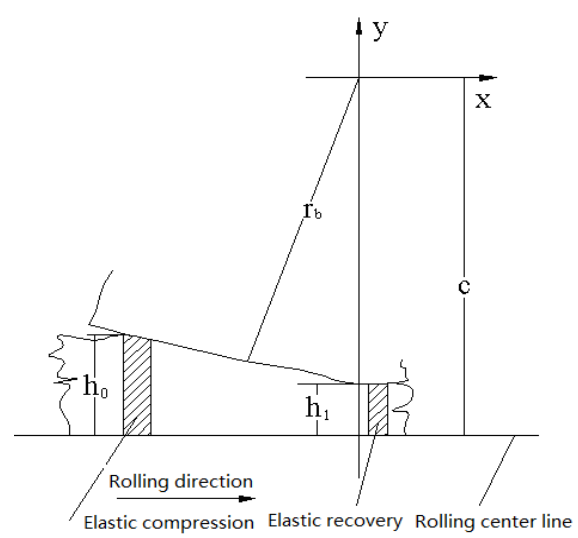

(b)

Figure 1: (a) Mechanical model and (b) deformation area of the rolling process. 
We make the following basic assumptions:

(1) All materials are homogeneous isotropic and keep invariant in the deformation process.

(2) The strip is elastic-plastic; it complies with Mises yield condition and Plandtl-Reass flow rules, and its width and thickness have a uniform size before rolling.

(3) The working roll and back-up roll are elastic bodies.

(4) In the deformation process, materials satisfy the law of constant volume.

(5) In the contact surface, the strip and rolls obey Coulomb linear friction law. When the friction force exceeds the yield shear limit, it will obey the shear friction theory, which is expressed as

$$
F / k=m
$$

where $F$ indicates the friction force, $k$ indicates the yield shear limit, and $m$ (a positive number less-than-or-equal-to 1) indicates the shear friction coefficient.

\section{Mathematical framework of the FM-BEM}

As commonly used numerical simulation methods, BEM and FEM are used to solve many engineering problems by forming a discrete matrix equation. The difference is that FEM forms a sparse stiffness coefficient matrix, while BEM forms a dense influence coefficient matrix.

\subsection{FMM formula}

Having originated from an electrostatic field, FMM [5-7] is used to compute the interactions between each two particles. A large number of particles are divided into different sets according to their spatial locations. When the distance between two sets is far enough, the interaction can be computed by the series expansion method, which can be expressed as:

$$
\Phi\left(X_{j}\right)=\sum_{\substack{i=1 \\ i \neq j}}^{n} \frac{q_{i}}{\left\|X_{j}-X_{i}\right\|}
$$

where $X_{1}, X_{2}, \ldots, X_{n}$ indicate points in $R^{3}$, , and $q_{1}, q_{2}, \ldots, q_{n}$ indicate a set of coefficients.

In a spherical coordinate system, an $n$-rank and $m$-order spherical harmonic function is defined:

$$
\begin{gathered}
Y_{n}^{m}(\theta, \phi)=\sqrt{\frac{(n-|m|) !}{(n+|m|) !}} \cdot P_{n}^{|m|}(\cos \theta) e^{i m \phi}, \\
P_{n}^{|m|}(x)=(-1)^{m}\left(1-x^{2}\right)^{m / 2} \frac{d^{m}}{d x^{m}} P_{n}(x)
\end{gathered}
$$


where $\phi$ and $\theta$ are the angle independent variables of the spherical coordinate, $P_{n}^{|m|}$ indicates an associated Legendre function, and $P_{n}(x)$ indicates an $n$-rank Legendre polynomial.

\subsection{GMRES algorithm}

For the solution of $\mathrm{N}$-order discrete system equations, the Gauss elimination method or related derivative methods are used in traditional BEM, and the order of magnitude is about $\mathrm{O}(\mathrm{N} 3)$. In the FM-BBEM, GMRES algorithm is introduced to solve the discrete system equations. Based on the Arnoldi $[8,9]$ algorithm, it is a newly developed iteration algorithm, and the order of magnitude is about $\mathrm{O}(\mathrm{N})$. Without forming a coefficient matrix, a series of recursive vectors are formed, which saves much storage space.

\subsection{FM-BEM expressions}

Suppose that a finite domain is expressed by $\Omega$ and its surface boundary is expressed by $\Gamma$, the boundary with known surface force is written as $\Gamma_{T}$, the boundary with a known displacement is written as $\Gamma_{U}$, and $\Gamma=\Gamma_{U}+\Gamma_{T}$. For elastic problems, the boundary integral equation without body force is expressed as follows:

$$
c_{i j} u_{i}(x)+\int_{\Gamma} T_{i j}(x, y) u_{j}(y) d \Gamma=\int_{\Gamma} U_{i j}(x, y) t_{j}(y) d \Gamma
$$

where $\boldsymbol{x}$ indicates an arbitrary point on the boundary $\Gamma, y$ indicates a source point, $\boldsymbol{c}_{i j}$ indicates a boundary shape coefficient, and the displacement $\boldsymbol{u}_{\boldsymbol{i}}$ and surface force $\boldsymbol{t}_{j}$ are solutions. In eq (4), $U_{i j}(x, y)$ and $T_{i j}(x, y)$ are Kelvin [10] fundamental solutions of displacement and surface force, respectively, which can usually be expressed as follows:

$$
\begin{gathered}
\boldsymbol{U}_{i j}(\boldsymbol{x}, \boldsymbol{y})=\frac{1}{16 \pi(1-v) \mu \boldsymbol{R}}\left[(3-4 v) \delta_{i j}+\boldsymbol{R},{ }_{i} \boldsymbol{R},{ }_{j}\right] \\
\boldsymbol{T}_{i j}(\boldsymbol{x}, \boldsymbol{y})=\frac{-1}{8 \pi(1-v) \boldsymbol{R}^{2}}\left\{\frac{\partial \boldsymbol{R}}{\partial \boldsymbol{n}}\left[(1-2 v) \delta_{i j}+3 \boldsymbol{R},,_{i} \boldsymbol{R},{ }_{j}\right]-(1-2 v)\left(\boldsymbol{n}_{j} \boldsymbol{R},,_{i}-\boldsymbol{n}_{i} \boldsymbol{R},{ }_{j}\right)\right\}
\end{gathered}
$$

where $\delta_{i j}$, a Kronecker function, is a two-order invariant tensor, $\boldsymbol{R}$ indicates the distance between an observation point and a source point, and $\boldsymbol{n}$ indicates the outer normal vector for the boundary $\Gamma$. The element coordinates are expressed as:

$$
x_{i}^{k}(\xi)=\sum_{l} x_{i}^{k l} \phi^{l}(\xi)
$$


In eq. (7), the summation term can be fast computed by the FMM. In order to make the fundamental solutions suitable for the $\operatorname{FMM}, \boldsymbol{U}_{i j}(\boldsymbol{x}, \boldsymbol{y})$ and $\boldsymbol{T}_{i j}(\boldsymbol{x}, \boldsymbol{y})$ are decomposed into the following expressions.

$$
\begin{gathered}
U_{i j}(x, y)=P_{i j}(x)\left(\frac{1}{R}\right)+Q_{i}(x)\left(\frac{1}{R} y_{j}\right) \\
T_{i j}(x, y)=W_{i j m}(x)\left[\frac{1}{R} n_{m}(y)\right]+S_{i m}(x)\left[\frac{1}{R} n_{m}(y) y_{j}\right]
\end{gathered}
$$

where$$
\boldsymbol{P}_{i j}(\boldsymbol{x})=\frac{\alpha}{2 \mu}\left[(3-4 v) \delta_{i j}-\boldsymbol{x}_{j} \partial_{i}\right]
$$$$
Q_{i}(x)=\frac{\alpha}{2 \mu} \partial_{i}
$$$$
\left.\boldsymbol{W}_{i j m}(\boldsymbol{x})=\alpha \mid(1-2 v)\left(\delta_{j m} \partial_{i}-\delta_{i j} \partial_{m}\right)-2(1-v) \delta_{i m} \partial_{j}+\boldsymbol{x}_{j} \partial_{i} \partial_{m}\right\rfloor
$$$$
S_{i m}(x)=-\alpha \partial_{i} \partial_{m}
$$

where $i, j, m=1,2,3, \partial_{i}$ indicates the partial derivative of $x_{i}$, and $\alpha=1 / 8 \pi(1-v)$.

Then eqs (8) and (9) are substituted into eq. (4), and eq. (4) can be discretized as follows:

$$
\begin{gathered}
c_{i j} u_{i}\left(x^{q}\right)+W_{i j m}\left(x^{q}\right) \sum_{k, l, s} \frac{1}{\left|x^{q}-y\left(\xi^{s}\right)\right|}\left\{u_{j}^{k l} \varphi^{l}\left(\xi^{s}\right) n_{m}\left[y\left(\xi^{s}\right)\right] J\left[y\left(\xi^{s}\right)\right] \omega^{s}\right\}+ \\
S_{i m}\left(x^{q}\right) \sum_{k, l, s} \frac{1}{\left|x^{q}-y\left(\xi^{s}\right)\right|}\left\{u_{j}^{k l} \phi^{l}\left(\xi^{s}\right) n_{m}\left[y\left(\xi^{s}\right)\right] y_{j} J\left[y\left(\xi^{s}\right)\right] \omega^{s}\right\}- \\
P_{i j}\left(x^{q}\right) \sum_{k, l, s} \frac{1}{\left|x^{q}-y\left(\xi^{s}\right)\right|}\left\{t_{j}^{k l} \phi^{l}\left(\xi^{s}\right) J\left[y\left(\xi^{s}\right)\right] \omega^{s}\right\}- \\
Q_{i}\left(x^{q}\right) \sum_{k, l, s} \frac{1}{\left|x^{q}-y\left(\xi^{s}\right)\right|}\left\{t_{j}^{k l} \phi^{l}\left(\xi^{s}\right) y_{j}\left(\xi^{s}\right) J\left[y\left(\xi^{s}\right)\right] \omega^{s}\right\}=0
\end{gathered}
$$

\subsection{Node-to-surface contact model}

The rolling process usually involves a strip's plastic flow and a big slip on the contact surface. The boundary condition has a nonlinear property. Nodeto-surface contact discriminant model is adopted, which is shown in Fig. 2. When two bodies $A$ and $B$ contact with each other, constraint conditions without penetration must be satisfied. The contact is discriminated by the coordinates of element nodes. After each incremental step, the position is 
known. Suppose that $P\left(x_{0}, y_{0}, z_{0}\right)$ is an arbitrary node on body $B$, and decides whether it contacts with element $S$ on body $A$ (the coordinates of element nodes are $X_{i}\left(x_{i}, y_{i}, z_{i}\right), i=1,2,3,4$, respectively). For the element $S$ with four nodes, arbitrary three nodes can determine a plane $\pi$, the distance from node $P$ to plane $\pi$ is $d$, and the projective point of $P$ is $P^{\prime}\left(x_{0}^{\prime}, y_{0}^{\prime}, z_{0}^{\prime}\right)$. If $d \leq \delta(\delta$ is a contact limit, and its default is $1 / 100$ of the length of minimal element) and $P^{\prime} \in S$, then node $P$ is determined to contact with element $S$, which means node $P$ is in coincidence with $P^{\prime}$. As the same time, the local coordinate $\left(\xi_{1}, \xi_{2}\right)$ of node $P$ in element $S$ can be computed by

$$
P^{\prime}=\sum_{l}^{M} X_{l} \varphi^{l}
$$

where $\varphi^{l}$ indicates the interpolating function, $\left(\xi_{1}, \xi_{2}\right)$ are independent variables, and $M$ indicates the number of element nodes.

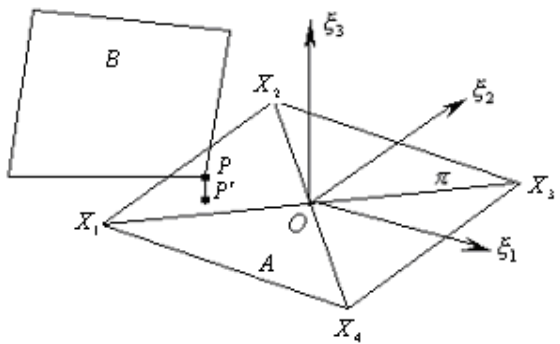

(a)

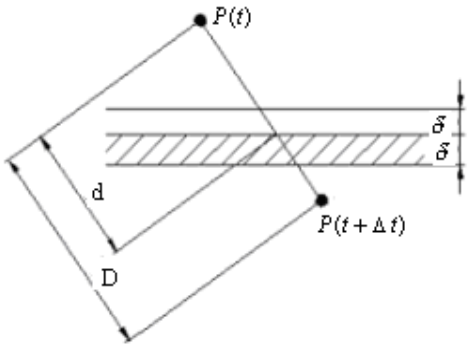

(b)

Figure 2: Node-to-surface contact model: (a) node-to-surface contact; (b) contact without penetration.

In each incremental loading step, $\delta$ can be used to detect whether contact or penetration occur. During the interval $[t, t+\Delta t]$, node $P$ moves from $P(t)$ to $P(t+\Delta t)$. If $P(t+\Delta t)$ is beyond the contact limit $\delta$, then penetration occurs and this incremental loading step must be subdivided. The subdivision rule is expressed as $\Delta t_{\text {new }}=\frac{d}{D} \Delta t_{\text {old }}$.

Numerical experiments show that the contact limit has a great effect on the computational accuracy and efficiency. A small limit is very helpful to the accuracy. But if it is too small, it will be very difficult to detect the contact of a node and an element, and a slightly larger step will make many nodes be treated as penetration. In order to avoid penetration, a smaller incremental load step can be selected. Once penetration occurs, the next step will be adjusted.

For the two deformation bodies in contact with each other, a bilateral contact check is carried out. In proper order, the contact bodies are checked to determine 
whether the probable contact nodes contact with some elements in other bodies and to distinguish possible penetrations. A bilateral contact check can greatly improve the computational accuracy, but it will take a lot of computing time and cause the increase of data storage.

\subsection{Mathematical programming for friction contact iteration}

Node-to-surface contact constraints show high nonlinearity, which makes the solution process very difficult and time-consuming. An effective measure is to linearize the nonlinear frictional contact constraints and establish an optimization mathematical programming model, which is suitable for large scale fast computations. The solution of the elastic-plastic problem is based on an incremental method. An appropriate loading step is very important for the convergence of computation. Too large a step will result in non-convergence, and too small a step will make the solution time very long. Because of the irreversibility of friction and plasticity, a step-by-step loading method is adopted for the displacement and surface force loads, which can improve the precision of solution. The number of loading step and the size of each step should be chosen according to the problem types and the load characteristics. Loading strategies have decisive influence on the computational accuracy and computational efficiency. So an appropriate loading strategy must be presented for the strip rolling simulation. The discrete equations are shown as below:

For the elastic body:

$$
\begin{array}{lc}
\text { For the elastic body: } & H^{i} \dot{u}^{i}=G^{i} \dot{t}^{i} \\
\text { For the elastic-plastic body: } & H^{i} \dot{u}^{i}=G^{i} \dot{t}^{i}+\Omega^{i} \dot{\sigma}^{p i} \\
& \dot{\sigma}^{i}=G^{i} \dot{t}^{i}-H^{\prime} \dot{u}^{i}-Q^{i} \dot{\sigma}^{p i}
\end{array}
$$

where, $i$ is the number of the body; $\dot{u}, \dot{t}$ are vector of the incremental displacement and surface force; $\mathrm{H}, G, H^{\prime}, G^{\prime}, \Omega, Q^{\prime}$ are the corresponding matrix respectively; $\dot{\sigma}, \dot{\sigma}^{\mathrm{p}}$ are stress and plastic stress increment in the plastic body.

\subsection{Network parallel computing}

In the rolling process, compared with the total surface of the roller, the contact deformation area is very small; only $6 \%$. To obtain the distributions of stress and surface force in the contact zone, meshes in the deformation zone must be subdivided. However, if the whole body is discretized according to the same subdivision criterion for the contact zone, the number of discrete elements will be too large. Therefore, in order to reduce the element number, the prolate elements with large length-width ratio (the maximum can reach 100) are used along the roller length direction. In this paper, an effective network parallel algorithm is used. Under PVM platform, parallel computing source programs are developed. Computing tasks are allocated to ten computers and computing results are collected in special sequence, which significantly improves the computational efficiency. 


\section{Simulation results and discussion for 2030 four-high mill cold rolling process}

The analysis of cold rolling processing with the strip width-thickness ratio being 1850 is a multi-body elasto-plastic contact problem with friction. By the FM$\mathrm{BEM}$, the computation time is 42 hours and 24 minutes, which is shorter than that by the FEM commercial software.

\subsection{Discrete model for 2030 strip mill cold rolling processing}

According to the symmetry of rolling mill and rolling processing, a 1/4 discrete model is used, as is shown in Fig. 3. The related computation parameters are shown in Table 1.
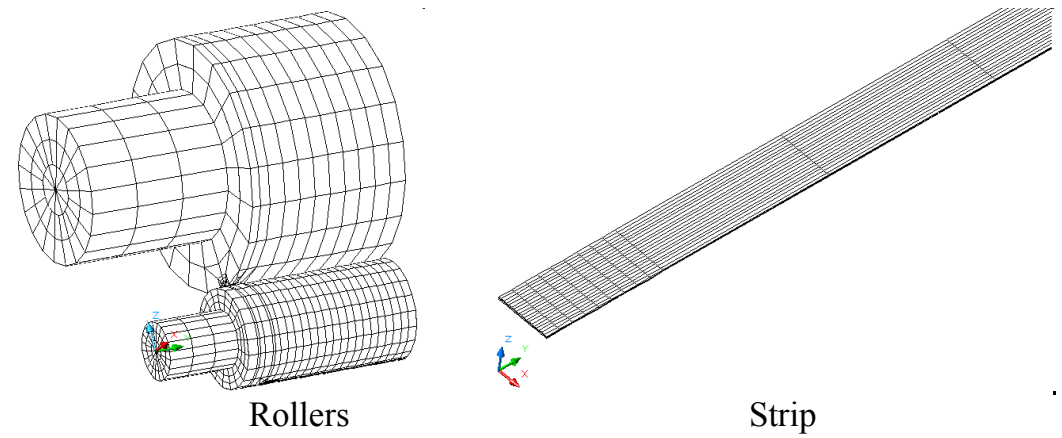

Figure 3: Discrete model.

Table 1: $\quad$ Rolling parameters.

\begin{tabular}{l|c|l|c|l|c}
\hline \multicolumn{2}{c|}{ Back-up roll } & \multicolumn{2}{c|}{ Working roll } & \multicolumn{2}{c}{ Strip } \\
\hline $\begin{array}{l}\text { Young modulus } \\
(\mathrm{E} / \mathrm{GPa})\end{array}$ & 210 & $\begin{array}{l}\text { Young modulus } \\
(\mathrm{E} / \mathrm{GPa})\end{array}$ & 210 & $\begin{array}{l}\text { Young modulus } \\
(\mathrm{E} / \mathrm{GPa})\end{array}$ & 206 \\
\hline $\begin{array}{l}\text { Poisson's ratio } \\
(\mu)\end{array}$ & 0.3 & Poisson's ratio $(\mu)$ & 0.3 & Poisson's ratio $(\mu)$ & 0.3 \\
\hline $\begin{array}{l}\text { Roller radius } \\
(\mathrm{R} / \mathrm{mm})\end{array}$ & 750 & $\begin{array}{l}\text { Roller radius } \\
(\mathrm{R} / \mathrm{mm})\end{array}$ & 300 & $\begin{array}{l}\text { Initial strip thickness } \\
(\mathrm{h} 0 / \mathrm{mm})\end{array}$ & 1.25 \\
\hline $\begin{array}{l}\text { Roll body length } \\
(\mathrm{L} / \mathrm{mm})\end{array}$ & 2030 & $\begin{array}{l}\text { Roll body length } \\
(\mathrm{L} / \mathrm{mm})\end{array}$ & 2230 & Strip width $(\mathrm{b} / \mathrm{mm})$ & 1850 \\
\hline $\begin{array}{l}\text { Friction } \\
\text { coefficient }(f)\end{array}$ & 0.1 & $\begin{array}{l}\text { Friction coefficient } \\
(f)\end{array}$ & 0.1 & $\begin{array}{l}\text { Friction coefficient } \\
(f)\end{array}$ & 0.08 \\
\hline $\begin{array}{l}\text { Contact limit } \\
(\Delta / \mathrm{mm})\end{array}$ & 0.001 & $\begin{array}{l}\text { Contact limit } \\
(\Delta / \mathrm{mm})\end{array}$ & 0.002 & Contact limit $(\Delta / \mathrm{mm})$ & 0.003 \\
\hline \multicolumn{1}{c|}{-} & - & - & - & Yield stress $\left(\sigma_{s} / \mathrm{MPa}\right)$ & 250 \\
\hline- & - & - & - & Stiffness coefficient $(\mathrm{H})$ & 0.002 \\
\hline \multicolumn{1}{c|}{-} & - & - & - & Reduction $(\%)$ & 20 \\
\hline
\end{tabular}


The hardening curve of yield stress for the strip material is shown in Fig. 4. Forward tension is applied according to the working condition.

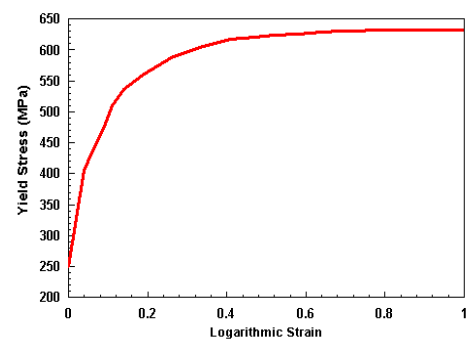

Figure 4: Hardening curve of the yield stress.

\subsection{Results and discussion}

In the contact deformation zone, the rolling pressure, longitudinal friction and transverse friction are shown in Fig. 5. Obvious peak value appears on the edge of rolling pressure, which is about $1000 \sim 1300 \mathrm{Mpa}$. The total rolling pressure is 1256 tons.

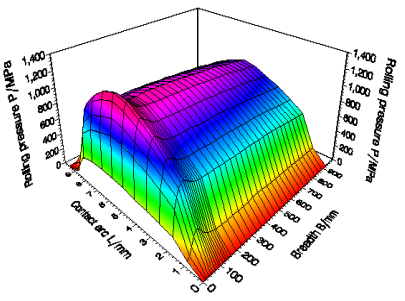

(a)

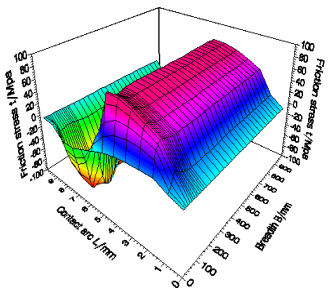

(b)

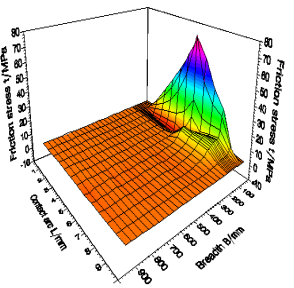

(c)

Figure 5: Surface force distributions in the contact zone: (a) rolling pressure; (b) longitudinal friction stress; (c) transverse friction stress.

In the deformation zone, transverse distribution of rolling pressure at the exit is shown in Fig. 6(a). The edge peak is especially obvious, which appears about $60 \mathrm{~mm}$ away from the rolled piece. Except the edge effect, the rolling pressure gradually reduces from the edge to the center. The reason is that the increasing of roll span results in the roll deflection significantly affecting the rolling pressure.

The distribution of rolling pressure along the center line of contact arc is shown in Fig. 6(b). The contact arc is $9.6 \mathrm{~mm}$ by actual measurement. Computed by the following equation

$$
l=\sqrt{R \Delta h}
$$

the length of contact zone is $8.66 \mathrm{~mm}$. In eq. (19), $R$ indicates the radius of working roll, and $\Delta h$ indicates the rolling reduction. The difference of computational and theoretical results explains the roller's elastic flattening phenomenon.

The distribution of longitudinal friction along the center line is shown in Fig. 6(c), which really represents the existence of forward and backward slip 
the contact zone and the friction force generated from slips. The transverse friction refers to the surface friction along the strip width direction. It violently changes at the edge because of the spread of resistance, and keeps gentle in the central area, which is consistent with the reality.

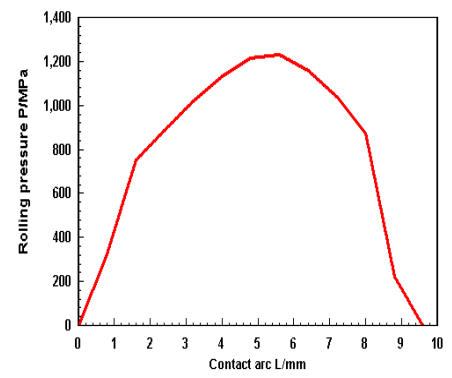

(a)

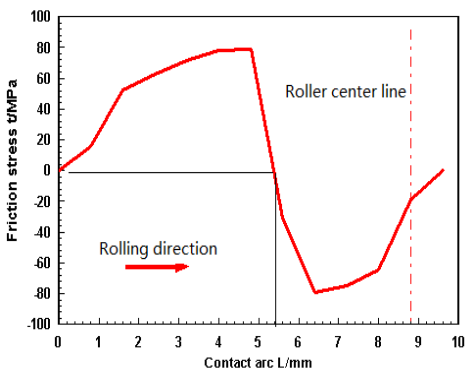

(b)

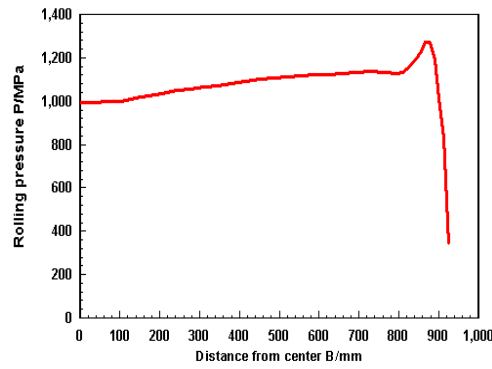

(c)

Figure 6: Distributions of the rolling force: (a) distribution of rolling pressure along the enter line; (b) distribution of longitudinal friction stress rolling along the enter line; (c) transverse distribution of rolling pressure along the exit.

As shown in Fig. 7, distribution of the friction force on the surface of rolled piece is given in vector mode. All the friction forces in forward and backward slip zones make in the same direction, namely, the neutral surface. Changes of friction direction resulting from width extrusion can be seen at the edge of the rolled piece.

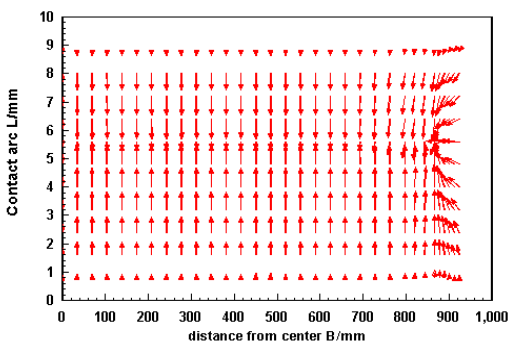

Figure 7: The surface friction force of rolled piece. 
The strip's metal flow condition in the deformation zone is also obtained. In the contact arc, the distribution of horizontal normal stress $\sigma_{x}$ along the thickness direction is shown in Fig. 8(a), where "+" indicates tensile stress and "-." indicates compression stress. The stress distribution is inhomogeneous, which shows the inhomogeneous deformation along the thickness direction. Being affected by the rigid end, the cross section at the entrance and exit in the deformation zone has tension/compression stress applied. Under the condition that the ratio of length and average thickness is 10 in the deformation zone, an inhomogeneous deformation phenomenon appears along the thickness direction. In the deformation zone, the distribution of metal velocity is shown in Fig. 8(b), where $V_{r}$ indicates the peripheral speed on the working roll surface, $V_{r x}$ indicates the component along its rolling direction, and $V_{x}$ indicates the metal velocity along the rolling direction.

At the thickness center of the deformation zone exit, the distribution of horizontal normal stress $\sigma_{x}$ along the width direction is shown in Fig. 8(c). Being affected by the roller's bending deformation, the distribution of horizontal normal stress along the width direction is inhomogeneous and the strip edge is pressed, which easily results in wave shape at the edge.

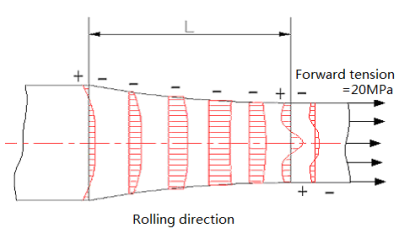

(a)

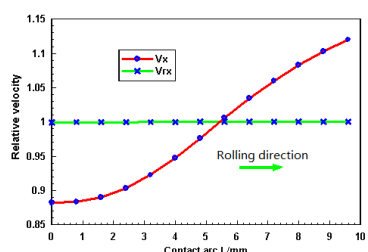

(b)

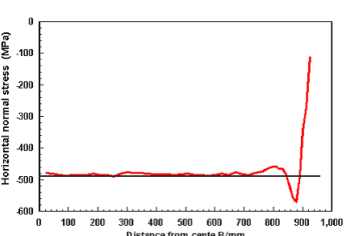

(c)

Figure 8: Velocity change in the deformation zone: (a) horizontal normal stress; (b) metal flow velocity; (c) horizontal normal stress.

The flattening and bending deformation of the working roll will directly affect the surface shape of the rolled piece and the stress distribution. The thickness distribution along the width direction at the strip exit is shown in Fig. 9. The strip shape takes on a thick middle and thin edge, and the thickness obviously reduces within about $80 \mathrm{~mm}$ away from the edge. The transverse thick difference reaches $0.0328 \mathrm{~mm}$, which is about $13 \%$ of the absolute reduction.

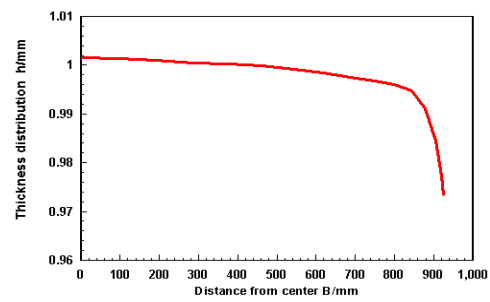

Figure 9: Transverse thickness distributions of the rolled piece. 
In the rolling process, the back-up roll is bent, and the working roll is flattened and deformed on the contact surface. Therefore, the profile can be controlled by changing the surface shape of the back-up roll. The surface force of the back-up roll and working roll in the contact zone is shown in Fig. 10. The transverse friction distribution is inhomogeneous. It is big at the edge and small in the middle, which shows the bending action of the back-up roll. The slip tendency at the edge is bigger than that in the middle. The whole contact zone is in the adhesive state, and no slip occurs. The length of contact arc between the back-up roll and working roll is $6.4 \mathrm{~mm}$. The pressure distribution in the contact zone is basically symmetrical.

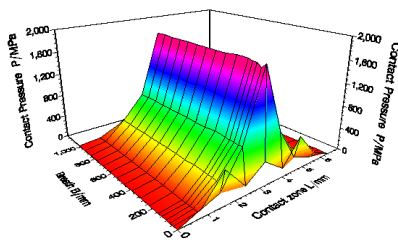

(a)

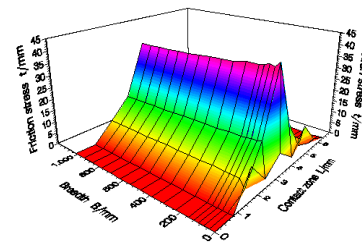

(b)

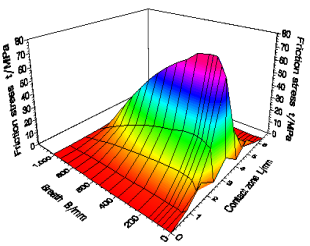

(c)

Figure 10: Contact surface force of the back-up roll and working roll: (a) contact pressure; (b) longitudinal friction; (c) transverse friction.

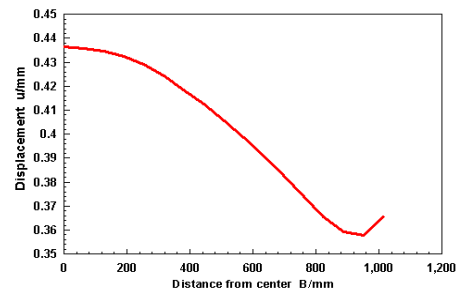

(a)

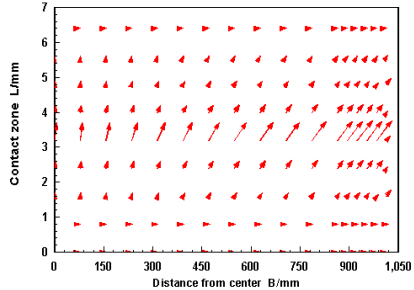

(b)

Figure 11: (a) Bending deflection and (b) friction force vector on the contact surface.

The distribution of longitudinal friction is similar to that of the contact pressure, the direction is consistent with the rotation direction of the back-up roll, and no relative slip occurs. The bending deflection of the back-up roll and the friction force vector on the contact surface are shown in Fig. 11. In the contact zone, the friction force distribution along the rolling direction is unilateral asymmetric, and it drives the rotation of back-up roll. Because the model is symmetric along the axial direction, the axial friction is symmetric and equal, which can cancel each other out.

\section{Conclusions}

(1) A review of three modern numerical solutions shows that the Finite Difference Method overly relies on artificial assumptions to obtain the desired results. The FEM is ineffective for the solution of large scale problems because it 
is difficult to set friction elements and the elements will dramatically increase when the 3D body is discretized, and the FM-BEM can be combined with parallel computation and used to efficiently solve the problem of cold rolling processing with a high width-thickness ratio.

(2) Some important parameters of strip rolling processing capacity are obtained, which include the rolling pressure, distribution regularities of the longitudinal and transverse friction stress, and the contact surface force. They are consistent with the actual measured distribution regularities. The rolling pressure takes on "pot bottom, cat's ear" shape, which is the most significant characteristic. The surface friction stress points to the neutral surface. They can be obtained from the velocity distribution or friction distribution on the strip surface. The two results are consistent, and the size of neutral angle fits well with the result from $\mathrm{S}$. Ekelund formula. The distribution of friction force vector clearly shows the metal flow state, the existence of forward slip, backward slip and stick zone, and the occurrence of width extrusion at the edge. The computational results represent the stress field with inhomogeneous deformation along the thickness and width directions in the strip rolling deformation area.

(3) The computational results show that the FM-BEM requires the least artificial assumptions and can give the most information. Compared with the FDM and the FEM, the FM-BEM has the highest precision.

\section{Acknowledgement}

The valuable advice of Emeritus Professor Junji Kihara from University of Tokyo, Japan, is very much appreciated.

\section{References}

[1] Q. H. Du. Numerical analysis method about the mechanical engineering of the continuous medium. The national conference about the finite element, Beijing, 352-359, 1978.

[2] Y. Saad and M. H. Schultz. GMRES: A Generalized Minimal Residual Algorithm for Solving Nonsymmetric Linear Systems. SIAM. J. Sci. Stat. Comput, 7(3): 856-869, 1986.

[3] Japanese Plastic Processing Society. Handbook of Technology of Plasticity. Corona Publishing Co., Ltd., 23-27, 2006.

[4] Jun Yanagimoto and Manabu Kiuti. 3-D coupling analysis of the sheet rolling processing by the Lagrange multiplier method of and 3-D rigidplastic FEM and 3-D elastic FEM. Plastic and Processing, (8): 1007-1012, 1991.

[5] V. Rokhlin. Rapid Solution of Integral Equations of Classical Potential Theory. J. Comput. Phys, 60: 187-207, 1985.

[6] L. Greengard and V. Rokhlin. A Fast Algorithms for Particle Simulations. J. Comput. Phys, 73: 325-34, 1987. 
[7] L. Greengard and V. Rokhlin. A New Version of the Fast Multipole Method for the Laplace Equation in Three Dimensions. Acta Numerical, 6: 229-270, 1997.

[8] M. H. Xu. The restarted GMRES method with appropriate parameters. Journal of Jiangsu Institute of Petrochemical Technology, 11(3): 52-55, 1999.

[9] B. J. Zhong, Z. S. Wang. An expansion of generalized conjugate gradient method of solving linear system of equations, Journal of Nanjing University of Aeronautics and Astronautics, 30(5): 515-520, 1998.

[10] Uhong Fu, Kenneth J. Klimkowski. A fast solution Method for ThreeDimensional Mary-particle problems of LinYear Elasticity. Int. J. Numer. Meth. Engng, 42: 1215-1229, 1998. 